\title{
The efficacy of antibiotics against Propionibacterium acnes biofilm infections on spinal implant material
}

\author{
Roger Bayston, Waheed Ashraf*, Rhys Clement, Robert Barker Davies, \\ Emily Tucker and Brian Freeman
}

\author{
Address: Division of Orthopaedics and Accident Surgery, School of Medical and Surgical Sciences, University of Nottingham. Queens Medical \\ Centre NG7 2UH UK \\ Email: Waheed Ashraf* - waheed.ashraf@nottingham.ac.uk \\ * Corresponding author
}

from 49th Annual Meeting of the Society for Research into Hydrocephalus and Spina Bifida

Barcelona, Spain, 29 June - 2 July 2005

Published: 30 December 2005

Cerebrospinal Fluid Research 2005, 2(Suppl I):S35 doi:I0.II86/I743-8454-2-SI-S35

\section{Background}

Propionibacterium acnes ( $P$. acnes), a common anaerobic skin commensal, has been implicated in delayed infection after implantation of spinal instrumentation. Biofilms of this organism have not been demonstrated previously. Spinal instrumentation is used to correct scoliosis in spina bifida.

We investigated the susceptibility of P.acnes growing within mature biofilms on titanium discs to Penicillin G alone, and to a combination with Rifampicin. Biofilms were exposed to therapeutic doses of antibiotics modelled on currently recommended treatment regimens.

\section{Materials and methods Biofilm group}

Surgical titanium discs ( $6 \mathrm{~mm}$ dia) were exposed ( $1 \mathrm{hr}$ ) to $P$. acnes, washed and immersed in fresh culture medium at $37^{\circ} \mathrm{C}$. The attached bacteria to titanium were allowed to mature for 6 days (biofilms). Subsequently, the discs were retrieved, sonicated and the number of viable bacteria counted by chemilumiluminesence and culture.

\section{Treated groups}

One group of mature biofilms was exposed to Penicillin G alone and the other with combination of Penicillin $G$ with Rifampicin for 96 hrs respectively. Again, the discs were retrieved, sonicated and the number of viable bacteria were counted by chemilumiluminesence and culture

\section{Results}

All P.acnes biofilms responded to treatment with a significant reduction in bacterial numbers. Combination therapy was more effective and produced greater reductions (96.6\%) of viable bacteria populating the biofilms than penicillin alone, $93.3 \%(\mathrm{p}<0.01)$. Complete eradication of the biofilm was not achieved in any cases.

\section{Conclusion}

P. acnes develop as a biofilm on spinal implant materials.

Antibiotic therapy significantly reduced the bacterial numbers. It remains to be determined whether longer periods of treatment will successfully eradicate the biofilm. 